\title{
Pierre Godeau
}

Né en 1930, le Professeur Pierre Godeau est décédé à Paris le 11 octobre 2018.

Sa carrière fut en tous points remarquable.

Interne des Hôpitaux de Paris à 23 ans, il gravit rapidement tous les échelons hospitalo-universitaires qui le conduisirent à acquérir une réputation internationale à la fois comme enseignant et comme chef d'école. Ses premières activités furent sous le signe de la cardiologie sous la direction de P. Soulié et Y. Bouvrain. Mais, c'est avec son maître, Fred Siguier, qu'il s'orienta vers la médecine interne dont il allait être le porte-drapeau universellement reconnu au sein du service qu'il dirigea, d'abord à l'hôpital Cochin, puis à la Pitié-Salpêtrière. Plus de 900 publications scientifiques parues dans des revues nationales et internationales recouvrent tout le champ de la médecine interne qui, perpétuant les principes hippocratiques, globalise l'approche diagnostique et thérapeutique en y intégrant les apports scientifiques modernes dans une vision humaniste où le patient reste l'objectif prioritaire.

Avec Claude Laroche et Michel Bourel, il créa la Société de Médecine Interne qu'il présida, jouant un rôle fédérateur national incomparable. Il fut à l'origine de l'autonomie et du développement de cette discipline actuellement individualisée au sein du CNU.

Cette activité de pionnier vit son couronnement dans la publication du Traité de Médecine, œuvre collective devenue pour chaque interniste la bible de référence. Il est remis régulièrement à jour par ses élèves, la dernière édition est en cours sous la direction de Loïc Guillevin.

La réputation de Pierre Godeau avait de loin dépassé nos frontières. Il était devenu le conseiller médical d'importantes personnalités de plusieurs pays francophones et effectuait de multiples missions d'enseignement à l'étranger. Son fils, Bertrand Godeau, chef de service à l'hôpital Mondor, perpétue l'engagement paternel en traçant le même sillon ininterrompu qui continuera à faire fructifier la médecine interne.

Pierre Godeau était membre de nombreuses sociétés savantes, entre autres la Société Médicale des Hôpitaux de Paris dont il fut Président avant d'être élu à l'Académie Nationale de Médecine. Il suivait attentivement les travaux de la Société de Biologie dont il était membre, confirmant l'intérêt qu'il portait aux recherches scientifiques.

Au-delà de la médecine, Pierre Godeau a développé ses talents d'écrivain dans trois ouvrages, l'un « Une aventure algérienne », consacré à son séjour militaire dans ce pays, le deuxième où il retrace sa carrière marquée par une vie familiale réussie, soulignant la pérennité de l'amitié, la force de la volonté pour résister aux caprices de la destinée. Le troisième ouvrage plus romancé relate comment un homme ordinaire peut devenir un assassin.

Pierre Godeau était un homme attachant, marqué par son élégance et son originalité vestimentaire.

Il était amateur de ski, de tennis et de natation; il pratiquait quotidiennement ces deux derniers sports à St Maur des Fossés où il résidait mais aussi en Corse où il avait une résidence secondaire. Il était passionné par les voitures anciennes dont il possédait plusieurs modèles.

Le professeur Pierre Godeau était Officier de la Légion d'Honneur, Officier de l'Ordre National du Mérite, titulaire des Palmes Académiques, de la Croix de la Valeur Militaire et Commandeur de l'Ordre marocain Ouissam Alaouite.

Tous ses proches conserveront le souvenir d'un ami fidèle, généreux, doué d'une ouverture d'esprit exceptionnelle et qui participa activement aux profondes mutations qui modernisèrent l'art médical dans la deuxième moitié $\mathrm{du} \mathrm{xx}^{\mathrm{e}}$ siècle.

Claude Jaffiol Président honoraire de l'Académie Nationale de Médecine 